\title{
A Data-driven Approach to Human-body Cloning Using a Segmented Body Database
}

\author{
Pengcheng Xi \\ National Research Council of Canada \\ pengcheng.xi@nrc-cnrc.gc.ca
}

\author{
Won-Sook Lee \\ University of Ottawa, Canada \\ wslee@uottawa.ca
}

\author{
Chang Shu \\ National Research Council of Canada \\ chang.shu@nrc-cnrc.gc.ca
}

\begin{abstract}
We present a data-driven approach to build a human body model from a single photograph by performing Principal Component Analysis (PCA) on a database of body segments. We segment a collection of human bodies to compile the required database prior to performing the analysis. Our approach then builds a single PCA for each body segment - head, left and right arms, torso and left and right legs - yielding six PCAs in total. This strategy improves on the flexibility of conventional data-driven approaches in $3 D$ modeling and allows our approach to take variations in ethnicity, age and body posture into account. We demonstrate our approach in practice by constructing models of a Caucasian male, an Asian male and a toddler from corresponding photographs and a Caucasian adult oriented database. We also discuss rapid consistent parameterization based on Radial Basis Functions (RBF) and non-optimization based learning systems to reduce execution time.
\end{abstract}

\section{Introduction}

The pioneering work of Blanz and Vetter [4] demonstrated that 3D models of a human face can be reconstructed from a single photograph and a 3D face database. This data-driven approach was also applied to full body reconstruction by Seo et al. [20]. However, a common disadvantage of all data-driven approaches is the difficulty in finding the parameters of an individual who does not belong to the data group. For example, can we reconstruct a child from a database of adults? Can we reconstruct an Asian out of a Caucasian database? Can we reconstruct a new body which has a slightly different pose from those in the database? In addition, the method used by Blanz and Vetter [4] and Seo et al. [20] has to solve the high dimensional optimization problem, which is a bottleneck in terms of calculation time and is vulnerable to falling into local minima.

In this paper, we introduce a new approach for reconstructing a 3D human body model from a single frontalview photograph. Our method is based on learning a relationship between the 3D meshes and their 2D silhouettes. We show that this relationship can be represented as a linear mapping. Once this mapping is established, a new 2D silhouette can be mapped to 3D space for a mesh reconstruction. This learning and then testing scheme works even better when it is applied to separated body segments. The main advantage of our method lies in its high efficiency and robustness in reconstructing subjects who are out of our training database either in ethnicity or in age. We demonstrate our results by reconstructing 3D bodies of a Caucasian male, an Asian male and a toddler from their corresponding frontal-view photographs. A few verifications are also conducted to support our conclusions.

\subsection{Literature review}

In this section, we focus on reviewing the work of face and body modeling, human shape variation, and finding mutually consistent surface representations.

DeCarlo et al. [6] did a representative work in the modeling of variation in human shape. The authors described a system capable of automatically generating distinct, plausible face geometries. This served as an early work in anthropometrical statistics for likely face measurements, which were later used for constraints on a parameterized surface.

In human modeling, generic model approach has been commonly used. Pighin et al. [17] achieved high realism in the synthesis of faces and facial expressions from photographs. Model fitting was used to adapt a generic model to fit an individual's face and facial expression. Using orthogonal photographs as input, Lee et al. [13] [12] success- 
fully applied geometric-based deformation to face and body modeling and animation.

Blanz and Vetter's work [4] was a milestone for human modeling and reconstruction making use of many generic models based on statistical analysis. It explored two key problems of computer-aided face modeling. First, new face images or new $3 \mathrm{D}$ face models could be registered automatically by making a large number of comparisons with an internal model. Second, the approach regulated the naturalness of the faces modeled and avoids those with an "unlikely" appearance. This work successfully introduced statistical approaches into computer graphics as a way of obtaining visual information from a given database.

One of the latest contributions to face modeling using a generic model came from "Head Shop" [9], which presented a versatile construction and deformation method for head models with anatomical structure, suitable for realtime physics-based facial animation. The work employed this technique to fit a generic head model to imperfect scan data and to simulate head growth from early childhood to adult age. Their deformation used volumetric radial basis functions (RBF). Though these approaches work well for convex objects, volumetric deformations are not suitable for entire bodies. Noh and Neumann [15] proposed another RBF-based approach, where dense surface correspondences were computed by volume morphing with RBF followed by a cylindrical projection.

Anguelov et al. [3] presented a framework for determining complex shape models from range scan data. Their framework consisted of algorithms based on the theory of probabilistic graphical models. This allowed complex shape models of different objects and object classes to be learned with minimal human intervention.

Whole-body parameterizations are more complicated because the human body does not easily lend itself to cylindrical mapping. Praun et al. [18] introduced an approach that built an n-way correspondence between meshes of the same topological type through the use of feature markers. This gave immediate correspondences between models and allowed remeshes to be performed. Their approach, however, could only be applied to complete surfaces. The parameterization would not work well on datasets in which some surface data was missing. Also, since this parameterization relied greatly on landmarks, problems arose when markers were missing.

A few more researches on body modeling appeared in the past several years. Lee [11] established consistent parameterization of human body shape from different kinds of inputs, e.g., a scanned data, an artistic model or a set of photographs, while the system needed a specially-structured generic model. Allen et al. [1] started from a subdivision template that resembled the range surface. They reparameterized the surface by sampling it along the tem- plate normals to construct a set of displacement maps. The matching technique used by Allen et al. [2] was based on an energy-minimization framework and is similar to that by Marschner et al. [14].

Seo and Magnenat-Thalmann [19] designed a body modeling system with anthropometric parameters. Starting from 3D data, they employed RBF to derive the deformation functions that generate the appropriate shape and proportion of body geometry. They applied a skeleton-driven approach to the rigid deformation of a generic model to target body surfaces. The latest work from Seo et al. [20] transferred this optimization scheme to reconstruction of human bodies from photographs. This method considered only silhouette information, but not the texture during model fitting. Park and Hodgins [16] used one hundred markers on a body to capture the surface information, where the main aim was to acquire skin deformation on animation frames.

\subsection{Our methodologies and contributions}

Figure 1 shows the outline of our methodologies. Our training process includes PCAs on consistently represented 3D body segments and PCAs on 2D silhouettes of these body segments. For certain body segment, i.e., the head, torso, left arm, right arm, left leg or right leg, we map its representations in all the training subjects onto the corresponding PCA space and thus form a coordinate matrix. Likewise, we map in 2D to get the segmented silhouettes their coordinate matrix. A linear regression is utilized to calculate a relationship matrix between the two coordinate matrices. This relationship is later used for testing on a new subject by projecting its silhouette onto segmented PCA spaces, mapping its coordinate in 2D PCA spaces to 3D PCA spaces for reconstructing 3D mesh segments. To make it clear, the 2D PCA space is built on contours of 2D silhouettes or their segments, and the 3D PCA space is based on $3 \mathrm{D}$ meshes or their segments.

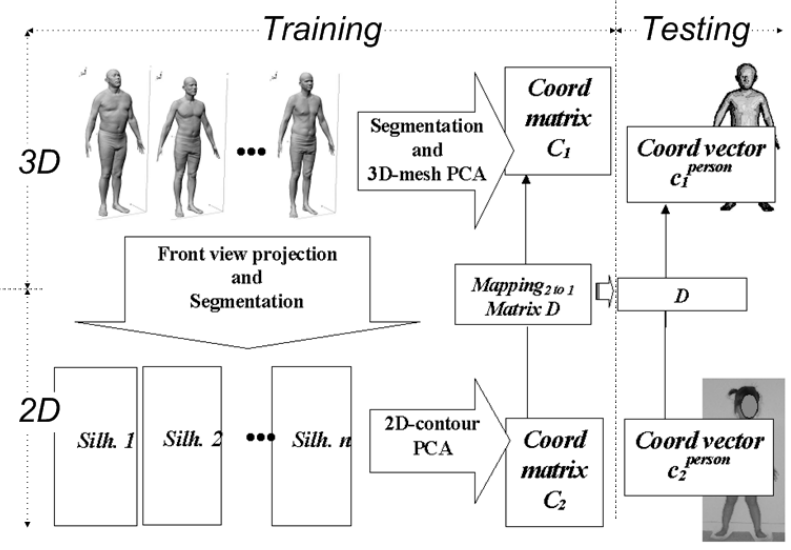

Figure 1. Outline of our human-body cloning 
The contribution of our work is threefold.

Segmented Body PCAs: We divide the body into segments (head, torso, two arms and two legs) and perform a separate PCA for each body part. This representation has a richer expressiveness than conventional PCA. It allows us to model Asians and children from a Caucasian adult database. The component-based approach has recently become popular in face recognition research. Several papers [8] [7] [10] [22] proved that component-based analysis raised the face detection and recognition rate over conventional whole-face recognition, especially on expressed faces.

Rapid Consistent Parameterization: We apply two steps of consistent parameterization to build morphable bodies from the non-morphable $3 \mathrm{D}$ meshes in the database. We perform feature-based parameterization using RBF, followed by surface-based parameterization using error minimization methods. We revise the definitions of weighted errors from previous work [2] and combine them to achieve same alignment quality with a higher efficiency. Our RBF-based error minimization method is twice as fast as the method by Allen et al. [2] and it yields less distortion after initial deformation.

Non-Optimization Based Learning System: From the work of Blanz and Vetter [4] [21] to the work of Seo, et al. [20], most of the approaches are based on minimizing a defined difference between the morphable 3D model and the target images. They lead to a high-dimensional minimization problem with two prominent disadvantages: low efficiency (a minimization problem of high dimensions) and undesirable local minima. We propose a learning scheme for the mapped coordinates in the 2D and 3D PCA spaces by first establishing consistent parameterization for both $2 \mathrm{D}$ contours of projected body images as well as $3 \mathrm{D}$ meshes. We then find coordinates of each body in the database by projection onto $2 \mathrm{D}$ and $3 \mathrm{D}$ principal components. A linear relationship between the mapped weight matrices along $3 \mathrm{D}$ eigenvectors and along 2D eigenvectors is found in the training step, which results in the instant calculation of the coordinates in 3D PCA space of an input photograph in the test step.

We organize the remainder of this paper as follows. The next three sections focus on the training step during which the database is analyzed. Section 5 describes the test step wherein the 3D mesh is built from an input photograph. The section 6 and 7 are devoted to verification and conclusion.

\section{RBF-based Consistent Parameterization}

Our approach operates on a high-resolution generic model with $328 \mathrm{~K}$ triangles. We use a two-step consistent parameterization, first purely landmarks-based and then surface-based for fast performance.

Radial basis function network greatly speeds up the alignment process. The RBF network [5] based on thinplate splines is entirely built on landmarks in both the generic model and target surfaces. We identify 73 anatomical landmarks on the generic model that correspond to those markers palpated through the skin on target surfaces in the CAESAR dataset. From our comparison of three radial basis functions (thin-plate, multi-quadrics and Gaussian), the Gaussian function is finally selected for our work as it shows the best performance in maintaining human-like shape after the rough deformation. RBF has several practical advantages. First, if the original generic model is significantly different from the target, the RBF can provide translation, rotation and scaling for the rough alignment in an efficient way. Second, the normal information of most vertices can be discarded because the transformed surface is already close to the target. Third, easier control on local deformation can be achieved by setting the number of influencing landmarks. [24]

In the second step, the fine mapping based on surface matching, marker information is discarded and the error definitions in [2] are simplified to two: (a) the difference between the source surface and target surface (data error) and (b) the difference between transformations on neighboring vertices (smoothness error). We use similar error minimization as was described in [25]. Compared to the three errors defined in [2], our work makes the control easier.

We find that, given enough time, the method from Allen et al. [2] approaches the level of accuracy reached by our method. By setting a threshold of the root mean squared error to be $0.006200 \mathrm{~m}$, our system consumes half the time on a PC with $3.1 \mathrm{GHz}$ CPU and 1GB memory.

\section{PCA Analysis on the Segmented Body Data}

The consistent parameterization establishes correspondences among models in the database. It allows us to perform PCA on the database, which leads to the characterization of the $3 \mathrm{D}$ shape variation from the most influential features to least influential ones. To enrich the PCA expressiveness in terms of different body ratios as well as postures, we use segmented body PCAs. The generic model is separated into six parts: head, left arm, right arm, torso, left leg and right leg. Neighboring segments have overlapping parts as displayed in Figure 2. This segmentation is directly applied to the consistently parameterized database to build six 3D-mesh PCA bases. 


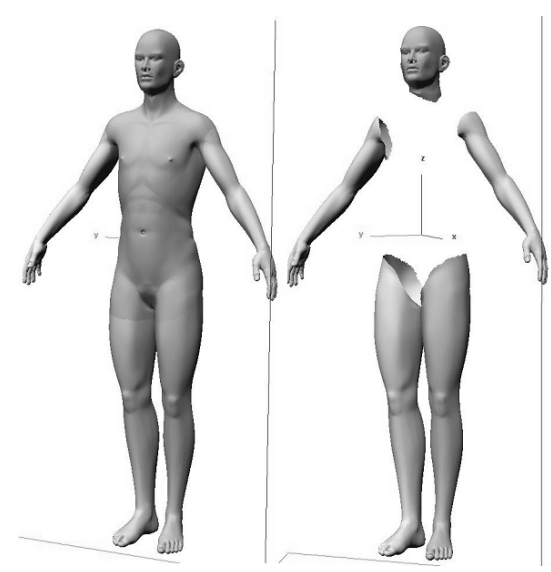

Figure 2. The segmented generic model

Figure 3 to 6 show enriched visible variations and when they are combined to form a whole body, the range of body shape variation is a lot wider than what we can achieve using non-segmented whole-body analysis, as shown in Figure 7. The CAESAR database is mainly composed of body scans of people from Europe and North America, where people have different body ratios from those of Asian people and young children. This analysis on segmented human bodies makes it possible to reconstruct people with different body ratios by first reconstructing body parts and then stitching them together for a complete body.

\section{2D-3D Relationship Learning}

\subsection{Consistent Parameterization of 2D- Contour}

To obtain the relationship between a 2D image (front view for this paper) and a $3 \mathrm{D}$ reconstructed body mesh, a consistent parameterization of a $2 \mathrm{D}$-contour of a projected image of each 3D mesh is required.

We obtain 2D projected images for all of the 3D meshes in our database by performing a perspective projection. Thereafter we need to find contours of each projected image and then attempt to make a consistent representation. By projecting a subset of all the 3D landmarks (on the shoulders, wrists, fingertips and so on) to $2 \mathrm{D}$, we are able to catch the markers on the silhouette automatically (using kd-trees to find nearest neighbors of these mapped markers on the contour as 2D landmarks) and build a consistent representation for each silhouette by contour tracking. We also obtain automatic segmentation of 2D silhouette into six body parts. There is overlapping between 2D neighboring contours as in the case of 3D mesh segmentation, but the overlaps can be found automatically by tracing backward and forward some pixels from certain feature points.

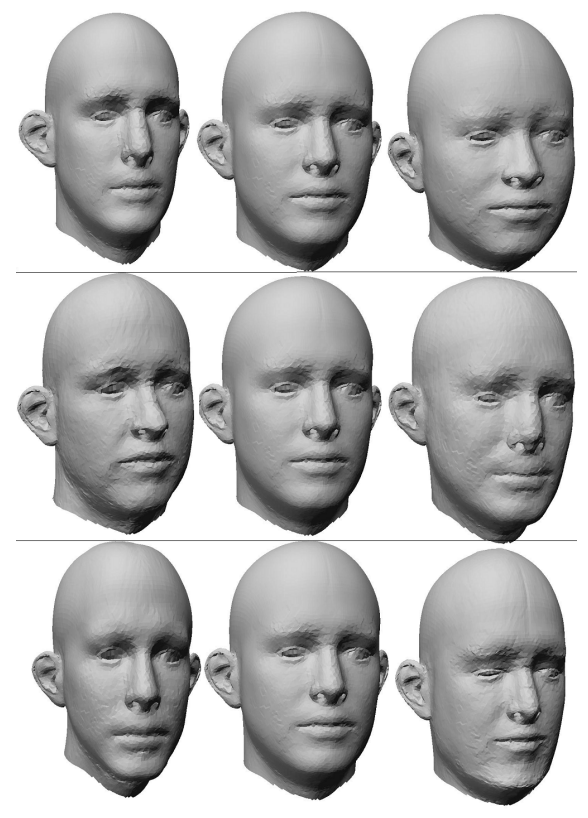

Figure 3. Mode variations along first three principal components of a head varying in: shape in the first row; facial features shape in the second row; facial feature ratio in the third row

\subsection{Mapping from 2D-Contour PCA Space to 3D-Mesh PCA Space}

After establishing consistent parameterizations on the 2D contour, PCA spaces are constructed. There are some common variations between the 2D-contour PCA and the 3D-mesh PCA spaces. Similar to the results in 3D mesh analysis, the first component corresponds to the height of the mapped body. The second variation represents a change in scale. The next six variations correspond mainly to weight and pose. The variations in $2 \mathrm{D}$ are a subset of those in $3 \mathrm{D}$ in that previous ones do not have the pose changes along the backward-forward directions. This observation presents a good basis in finding a relationship between the 2D and 3D PCA spaces.

After building PCA spaces for segmented 3D meshes as well as those for segmented 2D contours we find between them the mapping. Coordinates in the 2D-contour PCA space of an input photograph are then mapped to 3D-mesh PCA space to find the corresponding coordinates, thereby producing a 3D mesh of the input subject.

Since eigenvectors of the 3D-mesh PCA space have a significantly higher dimension than those of the 2D contour, it is difficult to find a direct relationship between them. Our training starts from projecting each $3 \mathrm{D}$ body mesh into the 3D-mesh PCA space and a matrix, $C_{1}$ (as shown in Fig- 


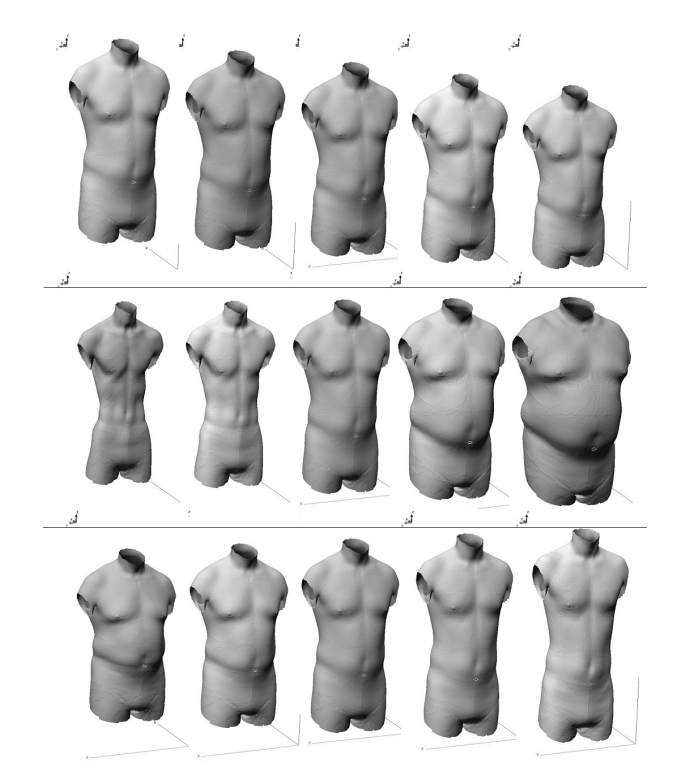

Figure 4. First three mode variations of the torso: height; weight, volume-preserving change. Those which are in the middle column are the averages.

ure 1), is formed where each column vector is the coordinates of each 3D body mesh in the PCA space. A similar process is followed in processing the $2 \mathrm{D}$ data and another matrix, $C_{2}$, is constructed likewise. We use linear regression to find a linear mapping from the 2D-contour coordinate matrix to the 3D-mesh coordinate matrix.

By mapping each 3D mesh onto the first $k_{1}$ principal components and forming the mapped vector into a matrix, we have the $\left(k_{1} \times m\right)$ coefficient matrix $C_{1}$, where $m$ is the number of 3D body meshes used for training. Similarly we obtain the coefficient matrix $C_{2}$ with a dimension of $\left(k_{2} \times m\right)$, where $k_{2}$ is the number of principal components maintained for the 2D contour PCA space.

Suppose a linear relationship $D$ between $C_{1}$ and $C_{2}$ is:

$$
C_{1}=C_{2} D
$$

$D$ can then be calculated by solving a minimum meansquared error problem (MMSE). The expression for $D$ is:

$$
D=\left(C_{2}^{T} C_{2}\right)^{-1} C_{2}^{T} C_{1}
$$

The matrix $D$ is thus a bridge between the 3D-mesh and the 2D-contour PCA spaces, where eigenvectors in each space are bases to this bridge. So for segmented mesh and contours, six matrices are built between 3D human body segments and their 2D contours.

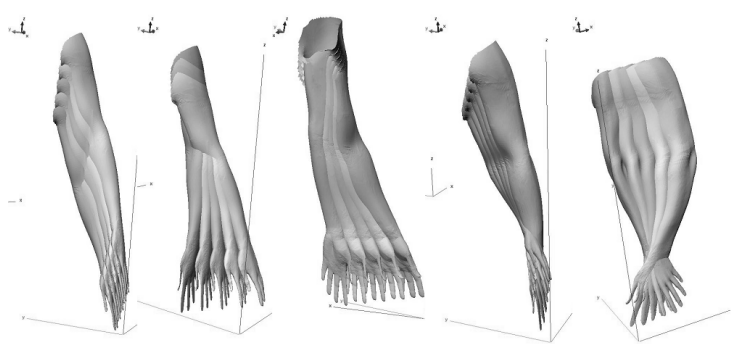

Figure 5. First five mode variations of a left arm: height; spreading extent; frontbackward pose; height of the upper-end of the arm; bending extent of the arm.

\section{A 3D Mesh from a Photograph}

\subsection{Shape Parameters of an Input Photo- graph}

Given an input photograph, the Sobel operator is used for edge detection to collect the silhouette information needed to form the body's contour. We place some landmarks to indicate that its contour is compatible with those from the database after applying global scaling and translation. Some manual interaction is possibly needed in this step depending on the images.

We map this contour into the 2D contour PCA space, which produces a vector composed of coordinates in the space, denoted as $c_{2}$ (as shown in Figure 1), having a dimension equal to the number of principal components maintained in 2D. Using the relationship defined in (2), we obtain the vector $c_{1}$, in 3D-mesh PCA space, composed of coordinates by:

$$
c_{1}=c_{2} D
$$

After obtaining the coordinate vector $c_{1}$, we can reconstruct the 3D body mesh by calculating a weighted sum of the principal components in 3D.

Figure 8 and Figure 9 shows the reconstructed bodies using the first 50 principal components in both 2D-contour and 3D-mesh PCA spaces in two cases: firstly, using a whole body and secondly, using a segmented body. The final reconstructed body from a segmented database is closer to the target image both in body ratio and on local shape. The improvement is more obvious in the case of the Asian male than the Caucasian male. The richer expressiveness of a segmented human body helps find a body when six segments are found independently to fit the input photograph.

Even though the toddler is a female, she does not have the typical woman's characteristics. We test various combinations between male database and female database. Fig- 


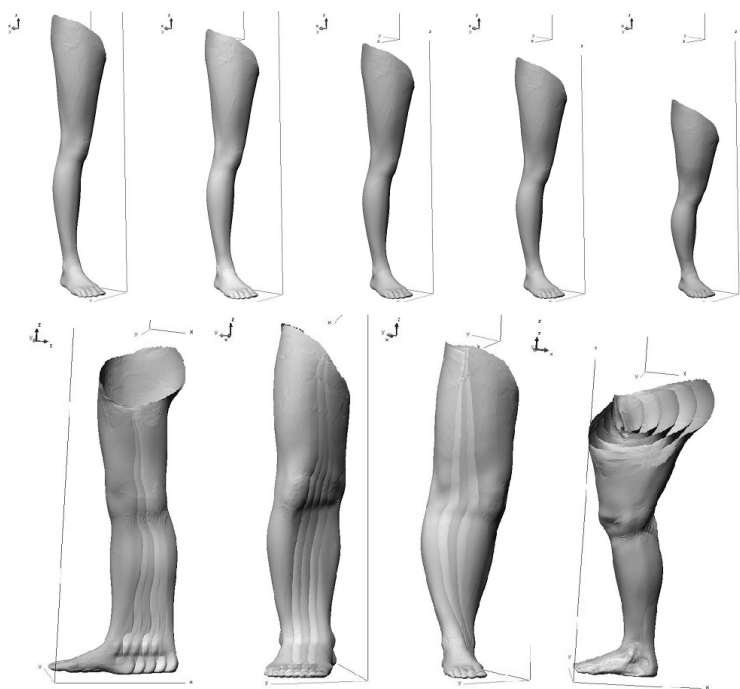

Figure 6. Upper five images show the variation along the first principal component for height of a right leg, while the lower four images show variations along the next four principal components: straightness of the leg; pose along left-right direction; bending extent; scale of the leg.

ure 10 depicts that the characteristics of the database influence the local shapes of the final reconstruction.

For each subject, there was an artifact on the hands in the non-segmented reconstruction if the arm pose differs greatly from that in the database. Here in segmented reconstruction, we find that the reconstructed segments maintain good shapes on the hands as well.

To get a continuous representation of the 3D body as the final result, we apply the RBF-based consistent parameterization, described in Section 2, with a generic model of resolution $100 \mathrm{~K}$ triangles to the reconstructed body segments where two error forces are used: smoothness and data. The resolution of the generic model in this step is lower than the one used for consistent parameterization in Section 2. It is to remove the artefacts between neighbouring body parts by smoothness force (as shown in Figure 11). The resultant continuous meshes are shown in Figure 12, where the diagonal views of the 3D meshes are also shown as well as the front views. The head shape of the toddler is unnatural due to her hair style. We are able to capture the approximated skull shape for an individual with either short hair or a ponytail as given in the CAESAR dataset. However, the toddler's hairstyle in the photograph is non-standard in the dataset, resulting in the unnatural head shape.

After obtaining a 3D model, we are thus able to get its texture by projecting the input photograph onto the model.

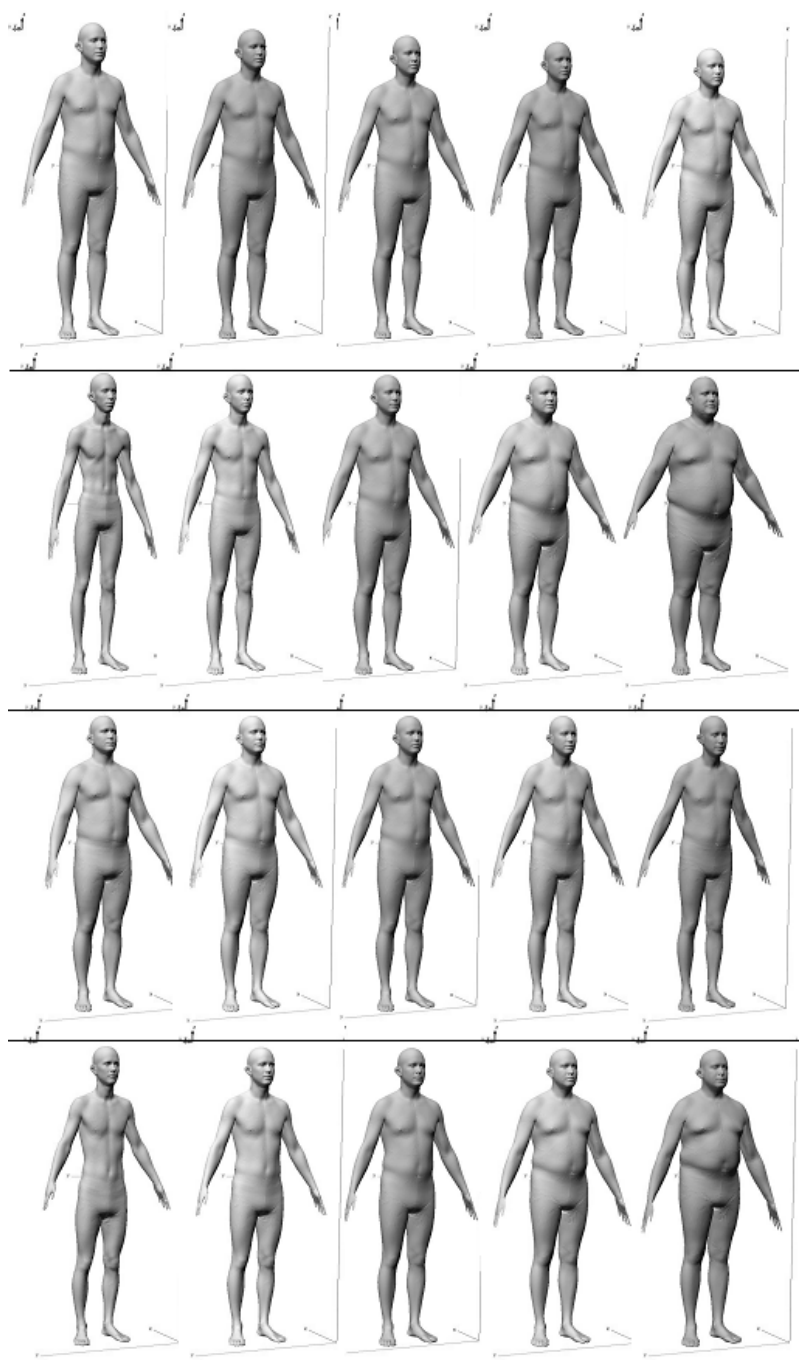

Figure 7. First four mode variations of a whole body: height; weight; posture; weight and body ratio.

The approach is from [12] and is automatic. We use front and back-view photographs to acquire the textures as shown in Figure 13. Since we had difficulty taking a back-view photograph of the toddler, we only map the front texture.

\section{Verification}

To justify the validity of our approach, we conduct a few more experiments on those CAESAR data, which have been withdrawn from our training process. Our testing examples include four Asian females, four Caucasian females, four African females, four Asian males, four Caucasian males and four African males [23]. For each datum, we project it and acquire its 2D silhouette with the same mapping con- 


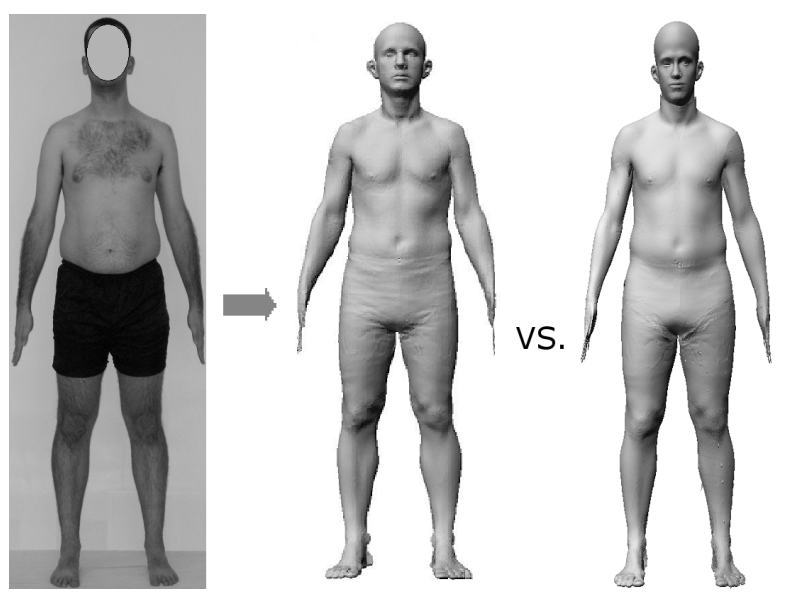

Figure 8. Reconstruction results for a Caucasian (from left to right): the input photograph, whole-body reconstruction result and segmented-body reconstruction result.

Table 1. Comparison of mean-error between wholebody and segmented-body reconstructions among different ethnic groups.

\begin{tabular}{|c|c|c|}
\hline \multirow{2}{*}{ Ethnic Group } & Whole-body & Segmented-body \\
\cline { 2 - 3 } & \multicolumn{2}{|c|}{ Mean Error } \\
\hline Asian & 0.0133395 & 0.001553 \\
\hline Caucasian & 0.0079353 & 0.0074499 \\
\hline
\end{tabular}

figuration as we used for training. Following the similar procedure as described in Figure 1, we reconstruct with the 2D contours their 3D shapes and are thus able to compare them with their original shapes.

For each testing mesh, we conduct both whole-body and segmented reconstructions. As we can see in Table 1, the mean error between the original mesh and reconstructed mesh for a Caucasian is almost the same for whole-body reconstruction and segmented-body reconstruction, while for Asian people, the segmented-body reconstruction makes great improvement over whole-body reconstruction. The number of samples used for the validation is not big, but it does provide a cue that the segmented-body reconstruction contributes to a more powerful reconstruction out of the scope of the database.

Besides the numerical validation, we also made visual comparisons between the whole-body reconstruction and the segmented reconstruction. Figure 14 demonstrates six sets of reconstruction results, ones from each ethnicity and gender group, shown in frontal and profile views.

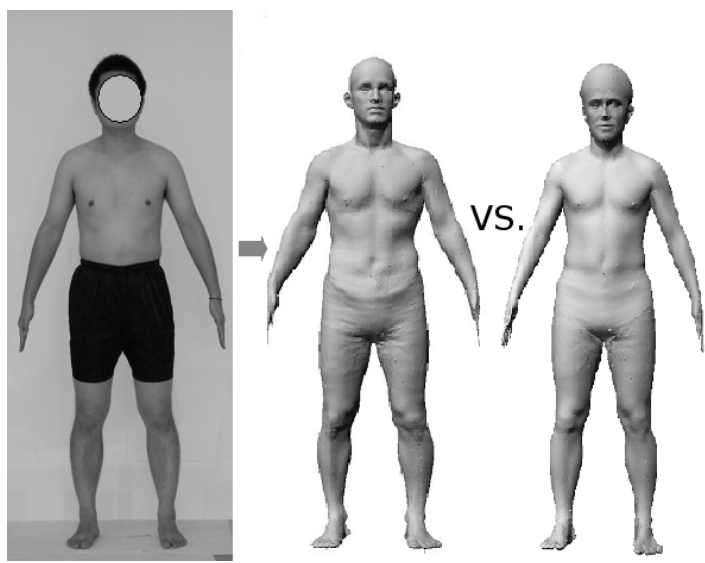

Figure 9. Reconstruction results for an Asian (from left to right): the input photograph, whole-body reconstruction result and segmented-body reconstruction result.

\section{Conclusion}

We have described a method for constructing a 3D body shape with a single photograph, focusing on covering a wider range of poses, ethnic and age groups of input human subject. Human subjects that do not belong to the ethnic or age group of the database are successfully reconstructed. Verification on subjects from different ethnicity and genders support that our subdivided PCA space based on a segmented body database demonstrates higher accuracy than that of whole-body reconstruction in data-driven modeling.

Our approach also avoids a high calculation cost and the local minima problems encountered in conventional optimization method for finding 3D shape parameters from an image. We apply 2D-contour PCA and 3D-mesh PCA and the training process learns matching between these two PCA spaces in order to find the parameters instantly without optimization in the test step. We also propose a two-step consistent parameterization method based on RBF which is two times faster than that of Allen et al. [2].

The database used for our experiment contains 964 males and 500 females. The longest calculation processes are the calculation of PCA with 3D meshes of 328K triangles, and mapping between 3D-mesh PCA and 2D-contour PCA, where both of them fully depend on the mesh resolution and database size. For 700 bodies, these two processes take about four hours on a PC with $3.1 \mathrm{GHz} \mathrm{CPU}$ and $1 \mathrm{~GB}$ memory. Note that these are off-line processes as they belong to the training step. The other on-site test processes combined take less than one minute to complete. 

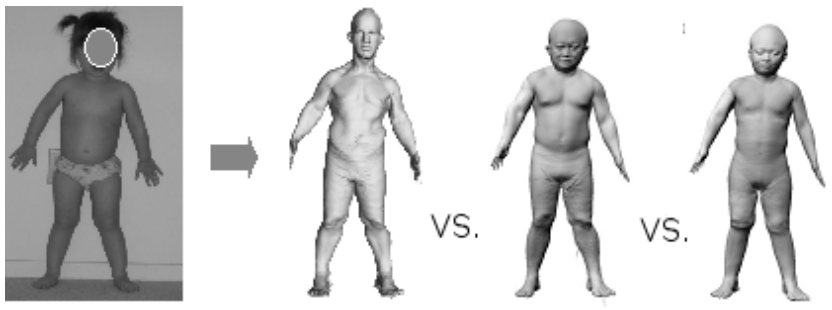

Figure 10. Reconstruction results for a toddler (from left to right): the input photograph; whole-body reconstruction result with a database of 964 male bodies; segmentedbody reconstruction result with a database of 964 male bodies; segmented-body reconstruction result with a mixed database of male and female bodies.

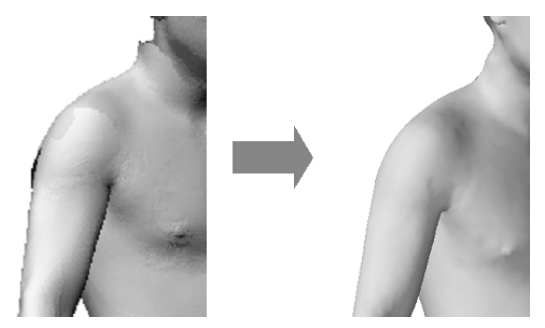

Figure 11. Knit the reconstructed mesh segments to form a continuous and smooth mesh.

\section{Acknowledgements}

The authors would like to thank Andrew Soon for polishing our work. We also express our gratitude to anonymous reviewers of our work.

\section{References}

[1] B. Allen, B. Curless, and Z. Popović. Articulated body deformation from range scan data. ACM Transactions on Graphics (ACM SIGGRAPH 2002), 21(3):612-619, 2002.

[2] B. Allen, B. Curless, and Z. Popović. The space of human body shapes: reconstruction and parameterization from range scans. ACM Transactions on Graphics, 22(3):587594, 2003.

[3] D. Anguelov, P. Srinivasan, D. Koller, S. Thrun, J. Rodgers, and J. Davis. Scape: Shape completion and animation of people. Transactions on Graphics (TOG), 24(3):408-416, 2005.

[4] V. Blanz and T. Vetter. A morphable model for the synthesis of 3d faces. ACM SIGGRAPH Computer Graphics Proceedings, pages 187-94, 1999.

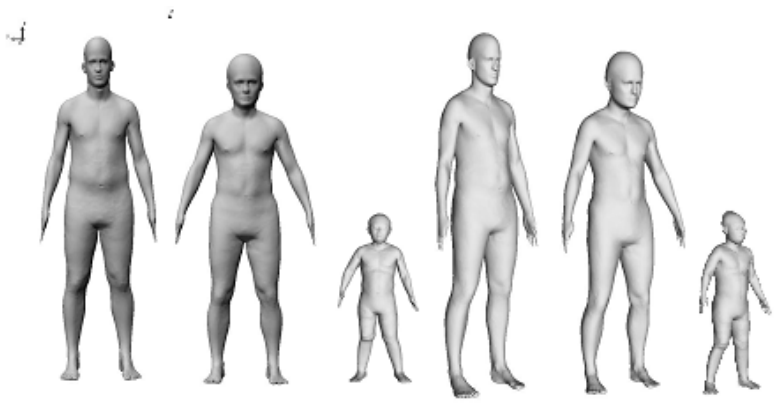

Figure 12. Final continuous reconstruction results for three subjects in their life-heights.

[5] F. L. Bookstein. Morphometric Tools for Landmark Data. Cambridge University Press, 1997.

[6] D. DeCarlo, D. Metaxas, and M. Stone. An anthropometric face model using variational techniques. ACM SIGGRAPH Computer Graphics Proceedings, pages 67-74, 1998.

[7] V. Franc and J. Matas. An extension of the componentbased lda descriptor by the generalized discriminant analysis. ISO/IEC JTC1/SC21/WG11 M8727, 2002.

[8] B. Heisele, T. Serre, M. Pontil, and T. Poggio. Componentbased face detection. Proc. IEEE Conference on Computer Vision and Pattern Recognition, 1:657-662, 2001.

[9] K. Kahler, J. Haber, H. Yamauchi, and H.-P. Seidel. Head shop: Generating animated head models with anatomical structure. Proceedings of the ACM SIGGRAPH Symposium on Computer Animation, pages 55-64, 2002.

[10] T.-K. Kim, H. Kim, W. Hwang, S. Kee, and J. H. Lee. Componentbased lda face descriptor for image retrieval. British Machine Vision Conference (BMVC), 2002.

[11] W.-S. Lee. Making one structured body. VSMM 2002, pages 558-567, 2002.

[12] W.-S. Lee, J. Gu, and N. Magnenat-Thalmann. Generating animatable $3 \mathrm{~d}$ virtual humans from photographs. Computer Graphics Forum (Proceedings of Eurographics 2000), 19(3):1-10, 2000.

[13] W.-S. Lee and N. Magnenat-Thalmann. Fast head modeling for animation. Image and Vision Computing, 18:355-364, 2000.

[14] S. Marschner, B. Guenter, and S. Raghupathy. Modeling and rendering for realistic facial animation. Proceedings of 11th Eurographics Workshop on Rendering, pages 231-242, 2000.

[15] J. Noh and U. Neumann. Expression cloning. Proceedings of the 28th Annual Conference on Computer Graphics and Interactive Techniques, pages 277-288, 2001.

[16] S. Park and J. Hodgins. Capturing and animating skin deformation in human motion. ACM Transactions on Graphics (Proceedings of SIGGRAPH 2006), 25(3):881-889, 2006.

[17] F. Pighin, J. Hecker, D. Lischinski, R. Szeliski, and D. Salesin. Synthesizing realistic facial expressions from photographs. Computer Graphics Proceedings SIGGRAPH 1998, pages 75-84, 1998. 

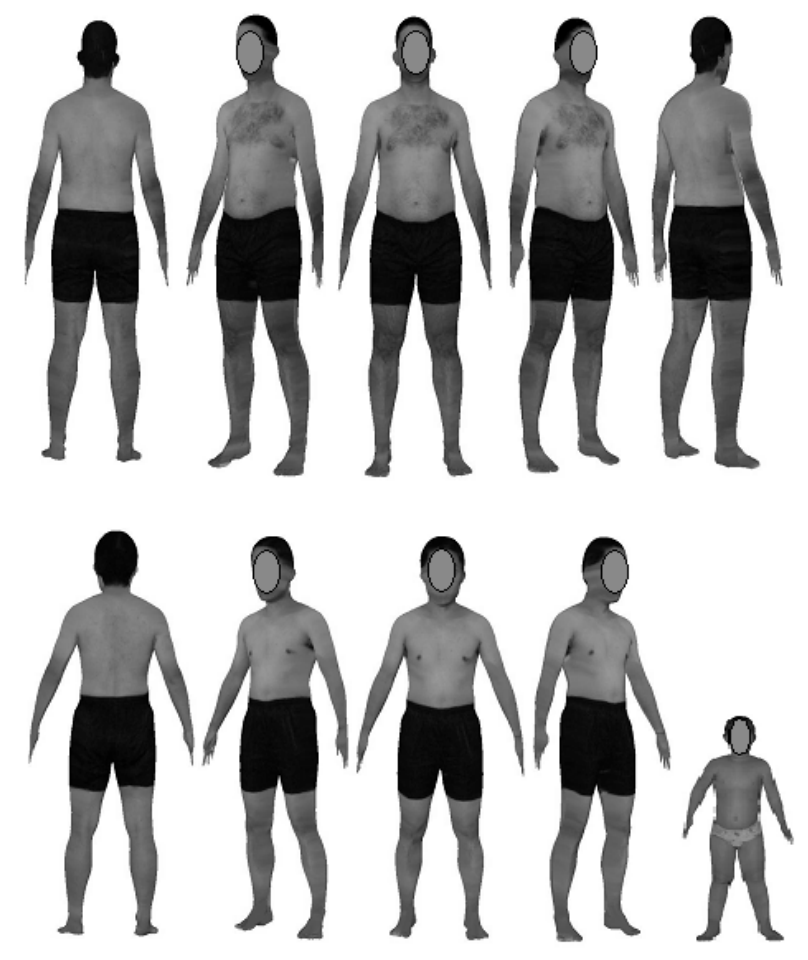

Figure 13. Textured 3D reconstruction results

[18] E. Praun, W. Sweldens, and P. Schroder. Consistent mesh parameterizations. Computer Graphics Proceedings SIGGRAPH 2001, pages 179-184, 2001.

[19] H. Seo and N. Magnenat-Thalmann. An automatic modeling of human bodies from sizing parameters. ACM SIGGRAPH 2003 Symposium on Interactive 3D Graphics, pages 19-26, 2003.

[20] H. Seo, Y. Yeo, and K. Wohn. 3d body reconstruction from photos based on range scan. Edutainment 2006, pages 849860, 2006.

[21] T. Vetter and V. Blanz. Estimating coloured 3d face models from single images: An example based approach. Proceedings of the 5th European Conference on Computer Vision, pages 499-513, 1998.

[22] B. Weyrauch, J. Huang, B. Heisele, and V. Blanz. Component-based face recognition with $3 \mathrm{~d}$ morphable models. IEEE Workshop on Face processing in Video, 2004.

[23] P. Xi. A pca-based approach to the $3 \mathrm{~d}$ reconstruction of human body from single frontal-view silhouette. Master thesis, University of Ottawa, 2007.

[24] P. Xi, W.-S. Lee, and C. Shu. Analysis of segmented human body scans. Proceedings of the Graphics Interface Conference, pages 19-26, 2007.

[25] C. Zhu, R. Byrd, P. Lu, and J. Nocedal. Algorithm 778. l-bfgs-b: Fortran subroutines for large-scale bound constrained optimization. ACM Transactions on Mathematical Software, 23:550-560, 1997.

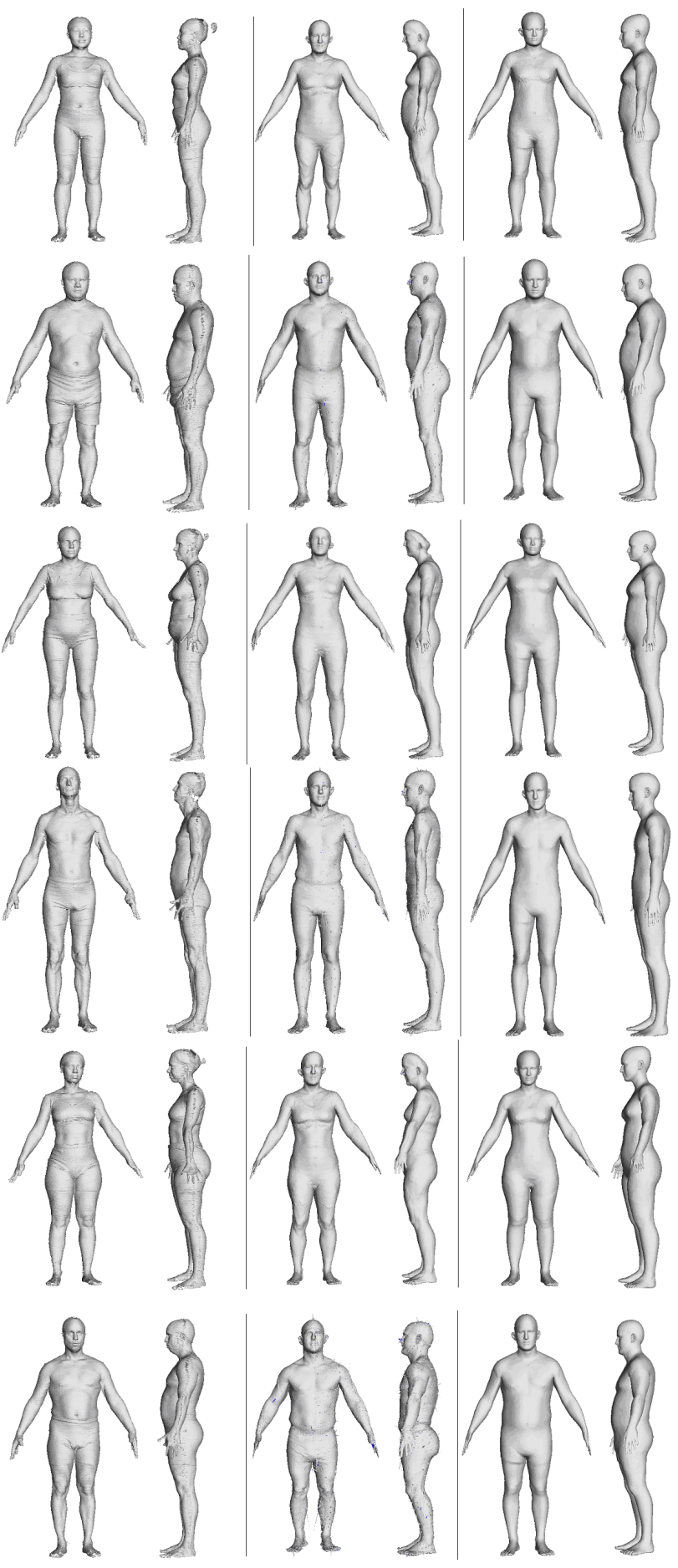

Figure 14. Comparison of the scan data, whole-body and segmented-body reconstruction results (from the first to the sixth row: Asian female and male, Caucasian female and male, and African American female and male; along each row, the first two are the original scans, the middle two are wholebody reconstruction and the right two are segmented reconstruction result) 